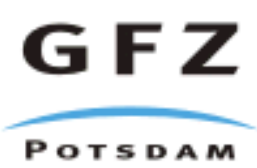

Originally published as:

Flechtner, F., Neumayer, K.-H., Kusche, J., Schäfer, W., Sohl, F. (2008): Simulation study for the determination of the lunar gravity field from PRARE-L tracking onboard the German LEO mission. - Advances in Space Research, 42, 8, 1405-1413

DOI: 10.1016/j.asr.2008.06.003. 


\title{
Simulation study for the determination of the lunar gravity field from PRARE-L tracking onboard the German LEO mission
}

\author{
Frank Flechtner ${ }^{*}$, Karl Hans Neumayer $^{1}$, Jürgen Kusche ${ }^{2}$, Wolfgang Schäfer ${ }^{3}$, Frank Sohl ${ }^{4}$ \\ ${ }^{1}$ GFZ Potsdam, c/o DLR Oberpfaffenhofen, Münchner Str. 20, D-82234 Wessling, Germany \\ ${ }^{2}$ GFZ Potsdam, Telegrafenberg, D-14473 Potsdam, Germany \\ 3 TimeTech GmbH, Curiestr. 2, D-70563 Stuttgart, Germany \\ ${ }^{4}$ DLR, Institut für Planetenforschung, Rutherfordstr. 2, D-12489 Berlin, Germany
}

Corresponding author: flechtne@gfz-potsdam.de

\begin{abstract}
A simulation study has been performed at GFZ Potsdam, which shows the anticipated improvement of the lunar gravity field model with respect to current (LP150Q model) or nearfuture (SELENE) knowledge in the framework of the planned German Lunar Explorations Orbiter (LEO) mission, based on PRARE-L (Precise Range And Range-rate Equipment Lunar version) Satellite-to-Satellite (SST) and satellite-Earth-satellite (SEST) tracking observations. It is shown that the global mean error of the lunar gravity field can be reduced to less than $0.1 \mathrm{mGal}$ at a spatial resolution of $50 \mathrm{~km}$. In the spectral domain, this means a factor of 10 (long wavelengths) and some 100 (mid to short wavelengths) improvement as compared to predictions for SELENE or a factor of 1000 with respect to LP150Q. Furthermore, a higher spatial resolution of up to $28 \mathrm{~km}$ seems feasible and would correspond to a factor of 2-3 improvement of SELENE results. Moreover, PRARE-L is expected to derive the low-degree coefficients of the lunar gravity field with unprecedented accuracy. Considering long mission duration (at least 1 year is planned) this would allow for the first time a precise direct determination of the low-degree tidal Love numbers of the Moon and, in combination with high precision SEST, would provide an experimental basis to study relativistic effects such as the periselenium advance in the Earth-Moon system.
\end{abstract}

Key words: Moon; Lunar gravity field determination; LEO; PRARE-L; Satellite-to-Satellite Tracking

\section{Introduction}

The precise knowledge of the lunar gravity field in terms of accuracy and resolution is crucial for understanding the internal structure and evolution of the Moon (e.g., Zuber et al, 1994). All currently available lunar gravity field models are mainly based on the analysis of insufficient radio tracking data of orbiting spacecraft, which include data from the Lunar Orbiter, Apollo, Clementine, and Lunar Prospector missions (Lemoine et al., 1997). Additionally, due to the synchronous rotation of the Moon around the Earth, all derived models lack information from the far-side. The most recent and probably best model available is the JPL Lunar Prospector mission result LP150Q (Konopliv et al., 2001) which is complete to degree and order 150 of a spherical harmonic representation. The model can resolve smallscale features up to $36 \mathrm{~km}$ half-wavelength with an approximate accuracy of about $30 \mathrm{mGal}$ on the near-side, but, due to the inhomogeneous input data which require strong constraints in the solution process, the model errors are definitely much larger (up to $200 \mathrm{mGal}$ ) on the farside (Wiezcorek, 2007). 
These deficiencies may be relieved with the Japanese SELENE (SELenological and ENgineering Explorer) mission, launched on September 14, 2007. SELENE consists of three satellites: a main satellite (MS), a relay satellite (RS) and a VLBI sub-satellite (VS), all three inserted in a coplanar orbit with an inclination of $90^{\circ}$. The MS has an initial $100 \mathrm{~km}$ altitude circular orbit controlled by attitude maneuvers, while the RS and VS are free flyers on inclined orbits with 100/880 km and 100/2400 km perigee and apogee, respectively. Three different observation types will be performed: First, 4-way satellite-to-satellite Doppler tracking (accuracy $1.0 \mathrm{~mm} / \mathrm{s}$ integrated over 10 seconds) between a ground station located in Usuda/Japan, the RS and the MS, directly enabling tracking of the MS by application of the high-low-satellite-to satellite tracking (HLSST) principle. As a result, the data gap on the farside can be filled for the first time. Second, differential VLBI tracking (accuracy $1 \mathrm{~mm}$ integrated over 100 seconds) between RS and VS will be used to precisely determine the RS orbit which serves as the reference of the 4-way Doppler measurements. Finally, 2-way Doppler measurements between RS and MS (accuracy $2.0 \mathrm{~mm} / \mathrm{s}$ integrated over 10 seconds) and between Usuda ground station and RS (accuracy $0.2 \mathrm{~mm} / \mathrm{s}$ integrated over 10 seconds) will help to stabilize the three-satellite orbit constellation. The overall goal is to derive an improved (one order of magnitude up to degree and order 30 with respect to LP100J, a precursor model of LP150Q) and homogeneous (incorporating data on the far-side) lunar gravity field up to degree and order 75 , where the long-wavelength part will be primarily observed by RS and VS and the shorter wavelengths by the MS. Further details can be found in Matsumoto et al. (2008).

Recently, a feasibility study of a Lunar Exploration Orbiter (LEO) has been initiated in Germany, in response to the conference "Exploration of our Solar System" held in Dresden/Germany in November 2006. LEO shall explore the Moon with unprecedented spatial and spectral resolution to significantly improve our understanding of the lunar surface composition, surface ages, mineralogy, physical properties, interior, thermal history, magnetic field, regolith structure, and gravity field. LEO would carry an entire suite of innovative, complementary technologies, including high resolution stereo camera systems, several spectrometers covering previously unexplored parts of the electromagnetic spectrum over a broad range of wavelengths, microwave and radar experiments, magnetometer and either one or two sub-satellites (Jaumann et al., 2007a; Jaumann et al., 2007b). A Phase-0 study has been finished in fall 2007 ending up with two different industry proposals which have been harmonized before entering Phase-A in April 2008. As a major outcome of this harmonization LEO and two identical sub-satellites are proposed to fly on a $50 \mathrm{~km}$ altitude orbit with $85^{\circ}$ inclination. The target launch date is September 2012, the mission lifetime for the main orbiter and the sub-satellites shall be 4 years.

To further improve the accuracy and resolution of the lunar gravity field as predicted by the SELENE mission, a low-low satellite-to-satellite (LLSST) experiment called PRARE-L (Precise Range and Range-rate Equipment - Lunar version) has been proposed by GFZ Potsdam and partners (TimeTech GmbH, Stuttgart; DLR Berlin; Technical University Berlin; University of Cologne; ZARM Bremen; and the University of Federal Armed Forces Munich). The general idea is based on the Earth gravity mission GRACE (Gravity Recovery and Climate Experiment) which provides time series of monthly gravity field solutions with unprecedented accuracy since April 2002 using K/Ka-band LLSST observations between a pair of twin satellites and HLSST GPS (Global Positioning System) observations for orbit determination and geo-location of the measurements (Tapley and Reigber, 2001). The LEO mission concept foresees that PRARE-L LLSST will be conducted between the identical subsatellites and that the information of HLSST GPS tracking (in case of GRACE) will be 
provided by PRARE-L SEST data. Details on the PRARE-L instrument are given in the next Section.
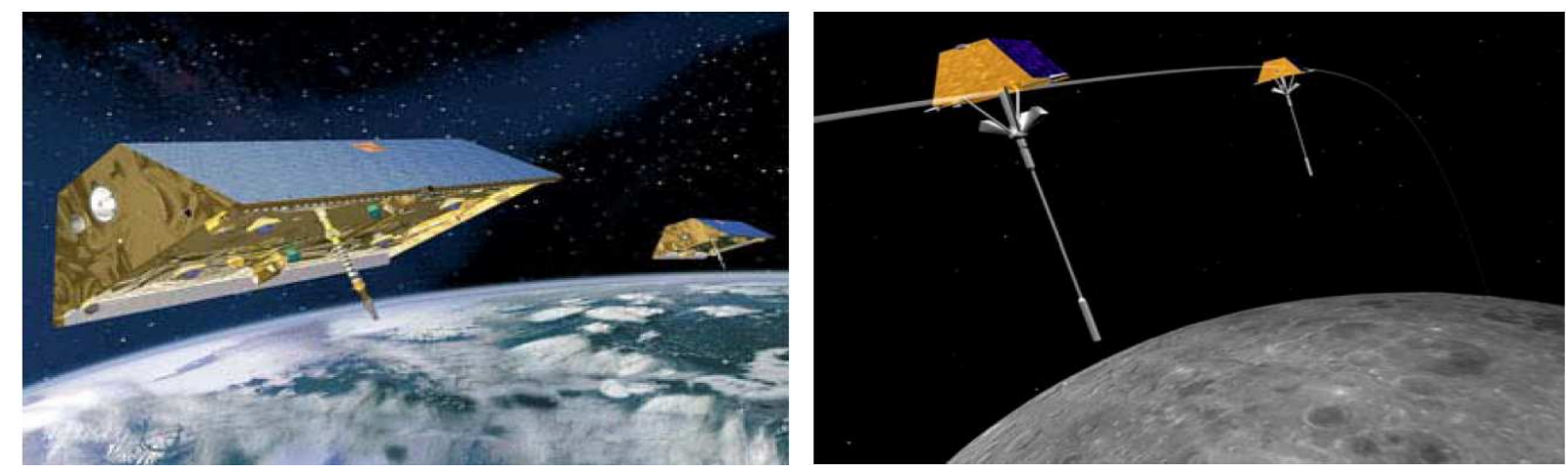

Fig. 1 GRACE (left) and LEO (right, in case of dual sub-satellite constellation, courtesy SpaceTech International, Immenstaad, Germany) twin satellite configuration for Earth and Moon, respectively, gravity field determination from LLSST observations.

A high accuracy and high resolution gravity field with a global mean error of less than $1 \mathrm{mGal}$ for half-wavelengths of $50 \mathrm{~km}$ (which was set as the science requirement for ESA's Moon Orbiting Observatory (MORO) mission, Foing, 1996) would allow to precisely

- determine the structure of the lunar crust by inversion of topography and gravity information,

- investigate the heterogeneity and elasticity of the lunar mantle,

- investigate the reason for the 1.9-km shift between the center of mass and center of figure of the Moon,

- improve the characterization of the size, physical state and composition of the lunar core or

- determine lunar Love numbers, which account for the tidal distortion of the Moon, for the first time precisely from time-varying gravitational perturbations

as well as to

- draw conclusions on the origin of the Moon and early Earth history,

- improve the orbit determination and maintenance of the LEO and all other historic and future lunar missions,

- investigate the Earth-Moon system as a whole and in detail the librations of the Moon, or

- conduct relativistic studies in the Earth-Moon system.

\section{The PRARE-L instrument}

PRARE-L is a further development of the Precise Range and Range-rate Equipment, which was designed and built completely in Germany and operated successfully between 1995 and 2007 onboard the ERS-2 (ESA Remote Sensing) satellite. PRARE is a fully autonomous space-borne, two-way, two-frequency (X- and S-band) tracking system with onboard data storage and central data processing. PRARE provided highly accurate range (15s normal point accuracy 1.5 to $3 \mathrm{~cm}$ ) and range-rate $(15 \mathrm{~s}$, integrated over 30 seconds, normal point accuracy 0.05 to $0.10 \mathrm{~mm} / \mathrm{s}$ ) measurements between the onboard instrument and a global network of mobile, autonomous ground stations used for precise ERS-2 orbit determination. The PRARE measurement signals are based on a combination of high frequency carriers (X-/S-band), appropriate pseudo-noise (PN) codes (10/1 MChips/s, and spread spectrum binary data $(2 / 4 / 10 \mathrm{~kb} / \mathrm{s})$. The signals are coherently derived from an ultra-stable quartz oscillator (USO) 
inside the space segment and disseminated by two dipole antennas. The ground stations, which are actually in view of the satellite, receive the X-band signal and demodulate the PNcode. Then, the PN-sequence is remodulated onto the X-band uplink and retransmitted to the space segment ("coherent, regenerative transponder") together with additional ground station calibration data to be applied during post processing. This includes the internal signal delay within the ground station as well as the differential delay between the primary X-band and the additional S-band downlink signals, which are used for ionospheric correction. The ground station design is fully coherent, i.e. the uplink carrier is in a well defined relationship to the received downlink carrier. The space segment can therefore measure the two-way range and the two-way Doppler shift of the signals very precisely by comparing the phase of the received signal to the phase of the transmitted signal, which is coherently derived from the onboard clock. These measurements can be carried out to up to four ground stations simultaneously. The overall accuracy of the system is mainly driven by the fully coherent two-way measurement principle, the high frequencies, and the appropriate resolution phase meters for code and carrier within the four space segment receivers. All generated tracking data as well as various corrective data are automatically collected and stored on-board together with housekeeping and calibration data. They are downloaded during each contact with the Command Station located in Stuttgart, Germany for further processing at the Master Station in Oberpfaffenhofen, Germany (Bedrich et al., 1997).

The PRARE design has already been upgraded to a Ku-band microwave link (MWL), with even higher signal chip rate (100 MChips/s), significantly improved carrier phase resolution of $60 \mathrm{fs}$ and lower transmit power $(0.5 \mathrm{~W})$. The instrument is to be used for space-ground frequency transfer at the level of $10^{-16}$ for time intervals from 1 day and longer for the ACES (Atomic Clock Ensemble in Space) experiment on-board the International Space Station ISS (Schäfer et al., 2007).

The lunar version of PRARE (PRARE-L) is based to a great extent on the PRARE and ACES-MWL tracking principle described above and will consist of two identical space segments which precisely measure the range-rate in Ka-band between two co-orbiting satellites separated by about $60 \mathrm{~km}$ with a resolution of about $1 \mu \mathrm{m} / \mathrm{s}$ (LLSST). Two-way ranging data between the satellites themselves and to a ground station on Earth (e.g. Weilheim, Germany) in X-band are available with an accuracy of better than $1 \mathrm{~m}$ (satelliteEarth-satellite tracking (SEST) or quasi-HLSST) at $\mathrm{cm}$-level precision. The ground station will have 2 independent receiver channels to distinguish between the two PN-coded X-band signals transmitted in parallel from both LEO satellites. As for PRARE/ERS-2, the clock synchronization will be performed by comparison of the onboard and ground clocks using a timing system at the ground station which compares the two transmitted 1 pps (parts per second) USO signals with a precise ground clock referenced to UTC.

The PRARE-L instrument uses the well-known principles of coherent communications to implement the following measurements and functions within a single package, all of them operating simultaneously:

- Tracking, Telemetry and Command,

- One- and two-way ranging using modulated carriers with full ambiguity removal,

- Doppler measurements using a suppressed carrier signal scheme,

- Two-way time- and frequency transfer between all elements, space and ground, and

- Separation between group- and phase delay measurements to detect plasma and plasma variations

Further potential applications include 
- Support of clock missions, where the clock's frequency acts as the gravity field sensor,

- Synchronization of clocks in space and between space and ground,

- Realization and use of a space-based time- and frequency reference,

- Transfering the high stability of ground clocks to space,

- Evaluation of one- and two-way ranging methods based on the precise knowledge of an on-board clock, and

- Demonstration of the feasibility of a coherent modulation scheme, which allows to obtain science data as well as to perform satellite operations simultaneously.

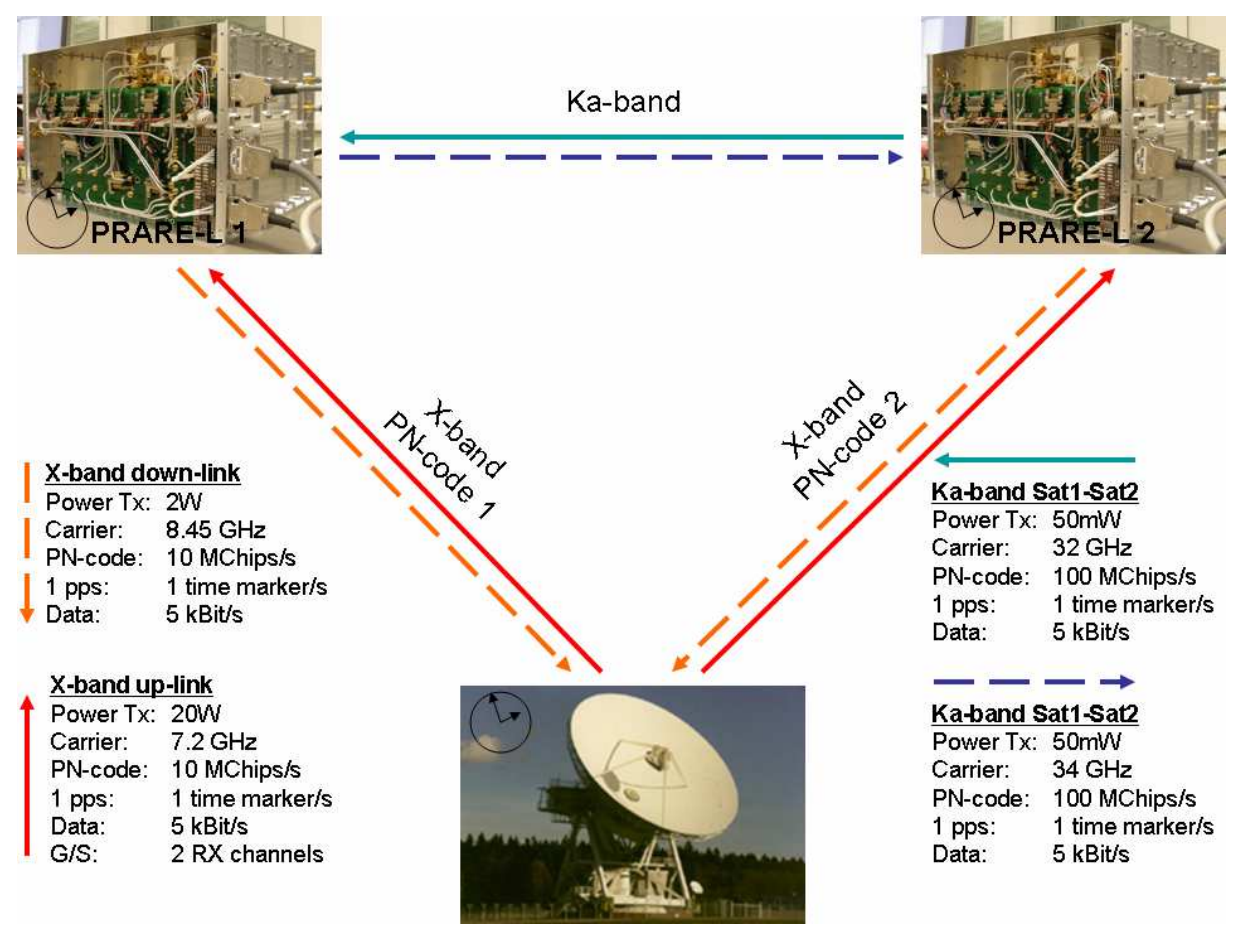

Fig. 2 Current PRARE-L signal link design (showing the ACES-MWL Engineering unit and the Weilheim 30m X-band receive antenna).

\section{Simulation Setup}

To examine how PRARE-L can improve the current knowledge of the lunar gravity field, a simulation study based on the classical dynamic approach including numerical integration, differential orbit correction and parameter adjustment, very similar to what is applied for GRACE data analysis (Reigber et al., 2005), has been performed by GFZ Potsdam within a phase- 0 study. The advantage of this method compared to analytical or semi-analytical approaches is the high accuracy and flexibility for the adjustment of parameters (which is in particular important in the event of data gaps). The only disadvantage is the relatively long computation time (the simulation and adjustment of a lunar gravity field up to degree and order 110 takes about 3 days on SUN VM890 used for GRACE data processing). All simulations were done with GFZ's EPOS (Earth Parameter and Orbit System) software relying on up-to-date IERS (International Earth Rotation Service) standards and background models, routinely used for the orbit and gravity field determination from tracking observations of CHAMP, GRACE and GOCE satellites, but updated to a special LUPOS (LUnar Parameter and Orbit System) version taking into account e.g. lunar tides and the lunar reference systems.

Within our simulation study the influence of various mission design parameters, such as orbital altitude and inclination, SST measurement accuracy and separation distance, Earth- 
LEO tracking accuracy, number of terrestrial ground stations or errors in the background models, such as solar radiation, on the lunar gravity field result have been investigated within realistic bandwidths ( $\mathrm{min} / \mathrm{max}$ values). The orbital altitude and inclination were chosen to 50 $\mathrm{km}$ and $85^{\circ}$, respectively, because this orbit guarantees (a) a nearly global coverage with only small polar data gaps and (b) a long lifetime ( 160 days) of the satellites without maintenance maneuvers necessary for an undisturbed collection of measurements necessary for gravity field determination. For a polar configuration, orbit maintenance would be required at a much higher frequency. The satellites are assumed to fly on a co-planar orbit with a separation fixed at $2^{\circ}(62 \mathrm{~km})$ which is the nominal angular GRACE distance value as a compromise between improvements of the long-wavelength (requiring a relatively large distance) and shortwavelength components of the lunar gravity field (resulting from smaller satellite separations). En echelon configurations with angular differences in the right ascension of the ascending node of the two satellites in addition to the nominal mean anomaly separation which could help to improve the (near-) sectorial components of the gravity field (Floberghagen, 1996) are not foreseen at present because of more complex flight dynamics and constellation control. Nevertheless, the present PRARE-L instrument design shall enable separation distances up to $4^{\circ}(124 \mathrm{~km})$ without decreasing the SST accuracy.

In a first step we simulated $5 \mathrm{~s}$ SST observations with a measurement noise of either $10 \mu \mathrm{m} / \mathrm{s}$ or $50 \mu \mathrm{m} / \mathrm{s}$, respectively, based on the LP150Q gravity field model, which is assumed as the "true" field, but truncated to degree and order 110 (corresponding to a half wavelength of 50 $\mathrm{km}$ ) to investigate the $1 \mathrm{mGal} @ 50 \mathrm{~km}$ science requirement as proposed in the MORO study (Foing, 1996) and also to save computing time. These SST noise realizations, both valid for a $5 \mathrm{~s}$ integration time, are 10 respectively 50 times larger than the pure PRARE-L measurement noise of $1 \mu \mathrm{m} / \mathrm{s}$ to take into account additional range-rate errors resulting from erroneous transformation of the SST observation from the phase center of the PRARE-L antenna to the center of mass at each of the two satellites. This could be caused e.g. by thermal effects, attitude errors, multipath at or movement of the high gain antenna or solar panel (in case of a main orbiter - single sub-satellite constellation). The factors of 50 and 10 are conservative and optimistic values valid at the beginning of phase-0. Meanwhile, after LEO concept harmonization and sub-satellite optimization at the beginning of phase-A, a factor not exceeding 3 appears realistic $(1 \mu \mathrm{m} / \mathrm{s} \mathrm{SST}$ measurement noise and $2 \mu \mathrm{m} / \mathrm{s}$ for center of mass transformation). The accuracy of satellite-Earth-satellite tracking (SEST), required for satellite orbit determination and SST selenolocation, has been simulated with 0.1 and $1 \mathrm{~m}$, respectively. The first value is a realistic assumption which can be achieved with a singlefrequency PRARE-L X-band ranging; the second value is a conservative upper bound. Additionally, a SEST accuracy of $5 \mathrm{~m}$ has been assumed in case that the tracking should not be performed with PRARE-L, but with standard X-band telemetry/telecommand (TM/TC) tracking. The number of Earth stations has been simulated as either one (DLR's (German Aerospace Center) existing 30-m antenna in Weilheim/Germany) or two (adding e.g. ESA's Deep Space Network (DSN) station in Perth/Australia). The data simulation period was chosen to be 28 days providing a closed orbit repeat pattern and thus enabling a solution of the lunar gravity field up to degree and order 150 (corresponding to a half wavelength of 36 $\mathrm{km}$ ). Other background models included are the Earth's gravity field (EIGEN-GL04C, maximum degree of 360 truncated at $\mathrm{n}=30$, Förste et al., 2007), JPL's planetary ephemerides DE405 (Standish, 1998), lunar tides (lunar Love number $\mathrm{k}_{2}=0.0216$ (Williams, 2007)) and solar radiation (8-hourly model adjusting 3 scaling factors per day, Floberghagen et al., 1996). A Moon albedo model has not yet been implemented in the LUPOS software. Therefore, the influence of albedo errors on the resulting lunar gravity field has to be studied in more detail in future simulations. 
In a second step, we solved the lunar gravity field up to degree and order 110 using the 28 days of simulated SST and SEST data (without gaps), but substituted the LP150Q model by a "clone", which was constructed by randomly corrupting the spherical harmonic coefficients with 1-sigma errors provided with the LP150Q model. This resulted in an initial lunar gravity field with a globally distributed mean error of about $36 \mathrm{mGal}$ and minima and maxima of about $150 \mathrm{mGal}$ which are similar to the values given in Wieczorek (2007). Additionally, the other parameters used in the first step have been analyzed within their minimum and maximum range (see Table 1). The corresponding lunar gravity field results are described in the following Section.

Table 1

Summary of simulation setup

\begin{tabular}{ll}
\hline Parameter & Value \\
\hline orbital altitude & $50 \mathrm{~km}$ \\
orbital inclination & $85^{\circ}$ \\
orbital eccentricity & 0 \\
satellite separation & $2^{\circ}(62 \mathrm{~km})$ \\
"true" lunar gravity field & LP150Q (up to $\mathrm{n}=110)$ \\
intial lunar gravity field for recovery & LP150Q corrupted by 1 -sigma errors (up to \\
& $\mathrm{n}=110)$ \\
Earth gravity field & EIGEN-GL04C (truncated at $\mathrm{n}=30)$ \\
planetary ephemeredes & DE405 $(\mathrm{JPL})$ \\
lunar tides & $\mathrm{k} 2=0.0216$ \\
solar radiation & $3 \mathrm{modeled}$ coefficients per day \\
SST measurement noise & $3 \mu \mathrm{m} / \mathrm{s}, 10 \mu \mathrm{m} / \mathrm{s}$ and $50 \mu \mathrm{m} / \mathrm{s}(5 \mathrm{~s}$ integration time) \\
SEST measurement noise & $10 \mathrm{~cm}$ and $1 \mathrm{~m}(\mathrm{PRARE}-\mathrm{L}), 5 \mathrm{~m}$ (TM/TC) \\
Earth ground station(s) & Weilheim/Germany $($ and Perth/Australia) \\
\hline
\end{tabular}

\section{Simulation Results}

The error spectrum of the retrieved lunar gravity fields has been analyzed by the coefficient sigma degree variance $\sigma_{n}$, which is defined as

$$
\sigma_{n}=\left[(2 n+1)^{-1} \sum_{m=0}^{n}\left\{\sigma^{2}\left(\bar{C}_{n m}\right)+\sigma^{2}\left(\bar{S}_{n m}\right)\right\}\right]^{1 / 2}
$$

where $\sigma\left(\bar{C}_{n m}\right)$ and $\sigma\left(\bar{S}_{n m}\right)$ are the sigma values of the spherical harmonic coefficients of degree $n$ and order $m$. No a-priori constraints such as Kaula's (1963) lunar rule of thumb of $3.5 \cdot 10^{-4} / \mathrm{n}^{2}$ had to be applied for the retrieval of coefficients. This means, that the PRARE-L instrument will provide a numerically stable and high-quality lunar gravity field within a time frame of only 28 days. Additionally, the PRARE-L results have been compared to SELENE predictions taken from Figure 3 of Matsumoto et al. (2008). The most interesting results, expressed in terms of coefficient sigma degree variances or gravity anomaly errors, for the different simulation scenarios of the previous Section, are described in the following.

Influence of SST measurement error

The influence of the SST measurement noise on the retrieved lunar gravity field, for the assumption that no orbit and no background model errors are present, is shown in Fig. 3 for the phase- 0 assumptions of $10 \mu \mathrm{m} / \mathrm{s}$ or $50 \mu \mathrm{m} / \mathrm{s}$. This scenario is certainly very optimistic but provides an upper bound for the lunar gravity field as derived from PRARE-L SST. Up to 
degree and order 75 (the anticipated maximum resolution of the SELENE mission) the coefficient sigma error degree variances for the $10 \mu \mathrm{m} / \mathrm{s}$ assumption are about 3-4 orders of magnitude below the LP150Q error level and still 2-3 orders of magnitude below the errors as predicted for SELENE. The resulting global mean gravity anomaly error (weighted Root Mean Square wRMS) is $0.10 \mathrm{mGal}$ with maximum values up to $0.76 \mathrm{mGal}$ (not shown). The result does not change if the Polar Regions, which are not observed by a $85^{\circ}$ inclined orbit, are not taken into account. In case of a very pessimistic $50 \mu \mathrm{m} / \mathrm{s}$ SST-uncertainty, the lunar gravity field result becomes more or less proportionally worse, resulting in mean and maximum gravity anomaly errors of $0.64 \mathrm{mGal}$ and $4.70 \mathrm{mGal}$, respectively. Consequently, the latest phase-A improvements down to $3 \mu \mathrm{m} / \mathrm{s}$ SST measurement noise will decrease this upper bound by a factor of 3 to global mean gravity anomaly error of about $0.03 \mathrm{mGal}$ (not shown).

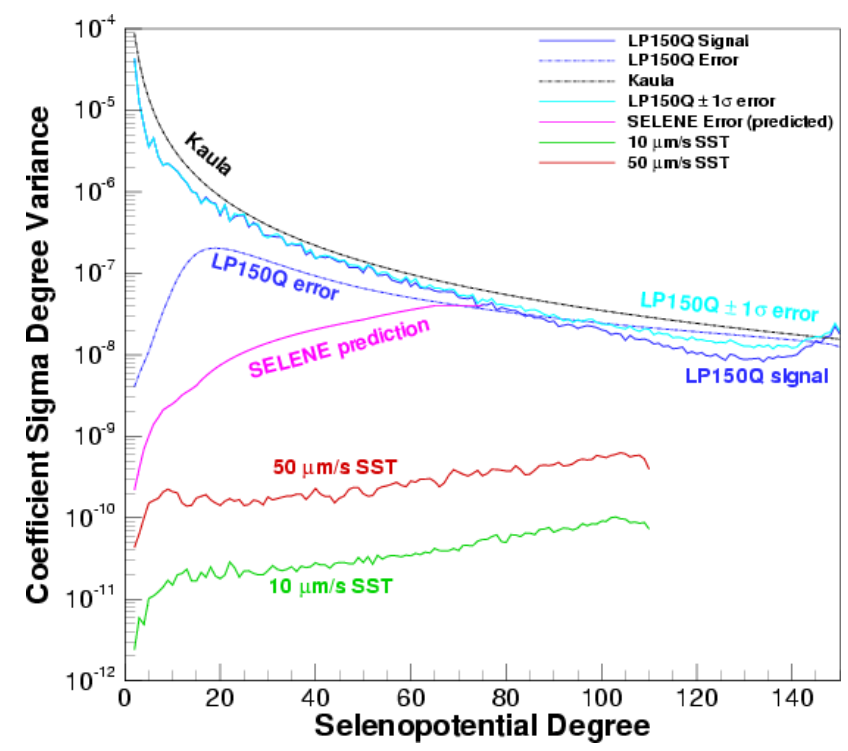

Fig. 3 Coefficient sigma signal degree variances for LP150Q (blue), LP150Q $\pm 1 \sigma$ (light blue) and Kaula' s rule of thumb (black) and coefficient sigma error degree variances of LP150Q (dashed blue), predicted for SELENE (purple), and for the phase- 0 assumption of a $10 \mu \mathrm{m} / \mathrm{s}$ (green) or $50 \mu \mathrm{m} / \mathrm{s}$ (red) PRARE-L SST uncertainty in the absence of orbital and background model errors.

\section{Influence of orbit errors}

In reality the satellite orbits will be determined by PRARE-L or TM/TC satellite-Earthsatellite tracking (SEST) from one or more ground stations. These observations are only available for the near-side and the resulting orbit accuracy is primarily depending on the SEST accuracy and the number of ground stations (or the number of SEST data gaps). Fig. 4 shows the results for the three SEST accuracy assumption $(10 \mathrm{~cm}$ and $1 \mathrm{~m}$ (PRARE-L) or $5 \mathrm{~m}$ (TM/TC)), one (Weilheim/Germany) or two ground stations (Weilheim, Perth/Australia) and a $10 \mu \mathrm{m} / \mathrm{s} \mathrm{SST-uncertainty} \mathrm{together} \mathrm{with} \mathrm{the} \mathrm{orbit} \mathrm{error-free} \mathrm{cases} \mathrm{for} 10 \mu \mathrm{m} / \mathrm{s}$ and $50 \mu \mathrm{m} / \mathrm{s}$ of Fig. 3. It becomes obvious that (a) the retrieved lunar gravity accuracy, especially for the long-wavelength part up to degree 20, becomes worse if orbital errors are introduced, (b) this decrease in accuracy does not depend on the number of tracking stations (see 1m SEST with and without Perth), (c) a $5 \mathrm{~m}$ SEST accuracy would further decrease the long wavelength information up to degree 30 and (d) due to the SEST-tracking and the thus increased orbit accuracy on the lunar near-side, the resulting gravity field has there a higher quality than on the far-side ( 0.10 respectively $0.23 \mathrm{mGal}$ global mean gravity anomaly error, not shown). 
Nevertheless, except for the very low degrees, all results are well below the $50 \mu \mathrm{m} / \mathrm{s} \mathrm{SST}$ accuracy quality, proving that the SST accuracy is the dominating factor to improve the lunar gravity field.

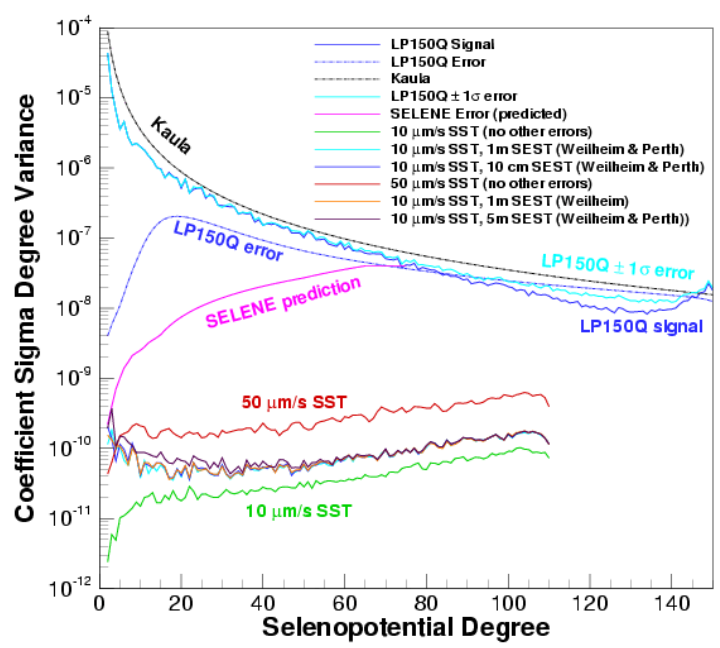

Fig 4. Coefficient sigma signal degree variances for LP150Q (blue), LP150Q $\pm 1 \sigma$ (light blue) and Kaula' s rule of thumb (black) and coefficient sigma error degree variances of LP150Q (dashed blue), predicted for SELENE (violet), and for the assumption of a $10 \mu \mathrm{m} / \mathrm{s}$ SST uncertainty and $10 \mathrm{~cm}$ (blue), $1 \mathrm{~m}$ (light blue) and $5 \mathrm{~m}$ (black) SEST to Weilheim and Perth as well as $1 \mathrm{~m}$ SEST to Weilheim only (orange). No background model errors have been introduced. For comparison the Fig. 3 result for a $10 \mu \mathrm{m} / \mathrm{s}$ (green) or $50 \mu \mathrm{m} / \mathrm{s}$ (red) PRARE-L SST uncertainties in absence of orbital and background model errors is shown as well.

\section{Influence of background model errors}

Besides SST and SEST errors also background model errors for e.g. direct solar radiation pressure, Moon albedo or lunar tides will influence the lunar gravity field result. Within this study only the influence of solar radiation has been investigated. A $10 \%$ fixed and nonadjusted error in the direct solar radiation pressure scaling coefficient can decrease the lunar gravity field accuracy by up to 1-2 orders of magnitude for the mid- and long-wavelength part, respectively. The largest errors result for the low degrees because the amplitude frequency spectra of solar radiation pressure induced orbit errors peak in the $0-2 \mathrm{cpr}$ (cycles per revolution) range, which in fact indicates that the radiation pressure, if not properly modeled, aliases in the low-frequency gravity field estimation (Floberghagen, 2002).

To test the influence of solar radiation errors the 8-hourly scaling factors, which are a-priori 1.0 during simulation of the PRARE-L observations, have been deliberately mismodeled by a random $\pm 10 \%$ error at the begin of the gravity field recovery for the $10 \mu \mathrm{m} / \mathrm{s}$ SST and $1 \mathrm{~m}$ SEST (only Weilheim) case and adjusted together with other unknown parameters (initial state, spherical harmonics). Fig 5 (left) shows the resulting signal degree variance for this case and for the case that no solar radiation errors have been introduced, proving that solar radiation can be sufficiently modeled without loosing signal in the recovered gravity field.

As has been mentioned above, the $10 \mu \mathrm{m} / \mathrm{s}$ SST assumption is no longer valid after subsatellite concept optimization at the beginning of phase-A. Therefore, Fig 5 (left) also shows the "solar-radiation case" for a $3 \mu \mathrm{m} / \mathrm{s}$ SST error. Additionally, the corresponding spatial 
difference between the "true" LP150Q and the recovered gravity field in terms of gravity anomalies is shown in Fig. 5 (right). It becomes obvious that the MORO scientific requirements (1 mgal @ $50 \mathrm{~km}$ half wavelength, (Foing, 1996)) for a static gravity field will be exceeded by a factor of 20 resulting in a global mean gravity anomaly error of $0.05 \mathrm{mGal}$ if only 28 days of PRARE-L SST and SEST data with $3 \mu \mathrm{m} / \mathrm{s}$ and $1 \mathrm{~m}$ accuracy, respectively, are analyzed, if only 1 ground station is (continuously) observing and solar radiation scaling factors are adjusted. The maximum errors of $0.34 \mathrm{mGal}$ will be obtained at the far-side, where no PRARE-L SEST observations for orbit determination will be available. As a result, the mean gravity error is expected to be $0.03 \mathrm{mGal}$ for the near-side and $0.07 \mathrm{mGal}$ for the far side. Note that the gravity anomaly error at the poles is below the maxima on the far side which implies that a $90^{\circ}$ inclined orbit is not necessary to get an optimal lunar gravity field. The homogeneous solution becomes also obvious when looking at the coefficient errors per degree and order depicted in Figure 6.
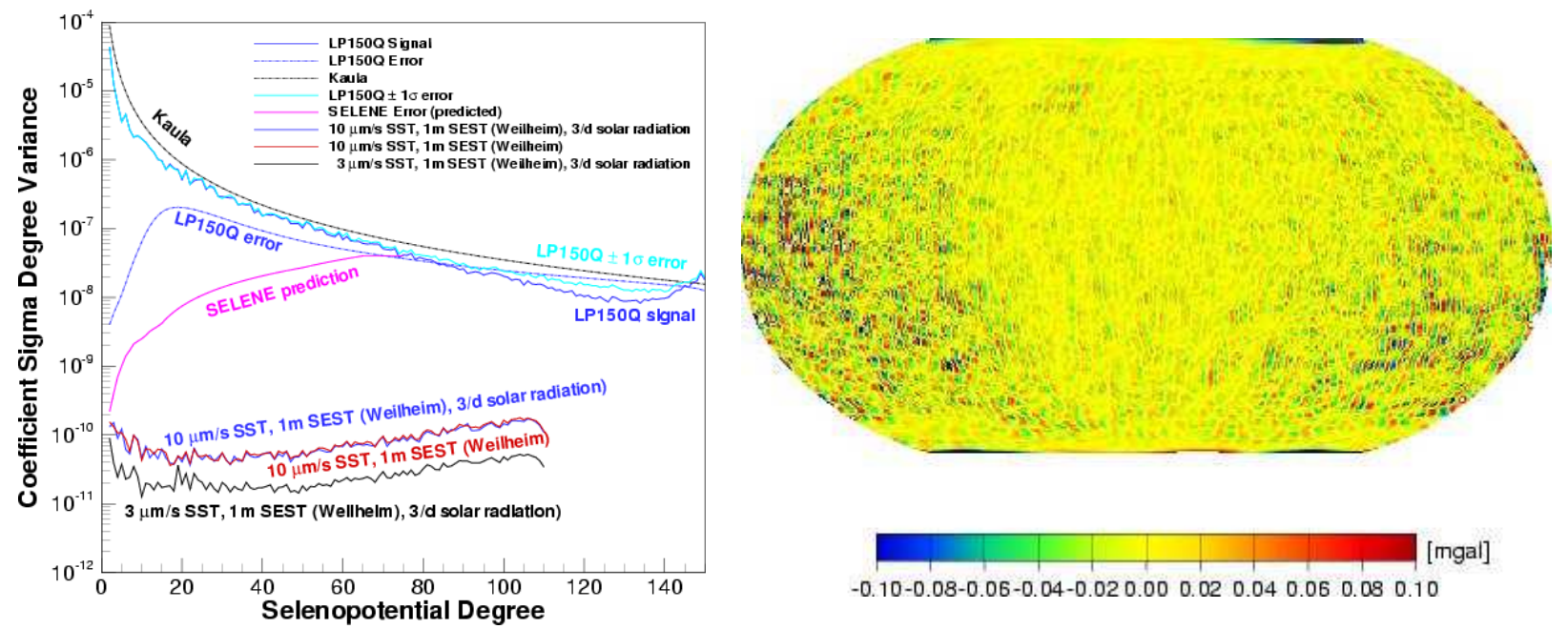

Fig 5. Left: Coefficient sigma signal degree variances for LP150Q (blue), LP150Q $\pm 1 \sigma$ (light blue) and Kaula' s rule of thumb (black) and coefficient sigma error degree variances of LP150Q (dashed blue), predicted for SELENE (violet), and for the assumption of a $10 \mu \mathrm{m} / \mathrm{s}$ SST uncertainty, $1 \mathrm{~m}$ SEST to Weilheim (red) as well as the same case but with additional adjustment of 3/d solar radiation scaling factors (blue). Additionally the result for $3 \mu \mathrm{m} / \mathrm{s} \mathrm{SST}$ uncertainty, $1 \mathrm{~m}$ SEST to Weilheim, and additional adjustment of 3/d solar radiation scaling factors is shown (black). Right: Difference between "true" LP150Q and retrieved lunar gravity field in terms of gravity anomalies for $3 \mu \mathrm{m} / \mathrm{s} \mathrm{SST}$ - and $1 \mathrm{~m}$ SEST-uncertainty from Weilheim tracking station only as well as for additional adjustment of 3/d solar radiation pressure scaling factors. Statistics (wRMS/min/max) for global difference [mGal]: 0.05/0.33/0.34; only near-side: $0.03 /-0.21 / 0.26$; only far-side: $0.07 /-0.33 / 0.34$; global, but without poles $\left(|\varphi|<85^{\circ}\right)$ : $0.05 /-0.33 / 0.34$. The near-side of the Moon is in the center of the figure, the far-side on the left and right, respectively. 


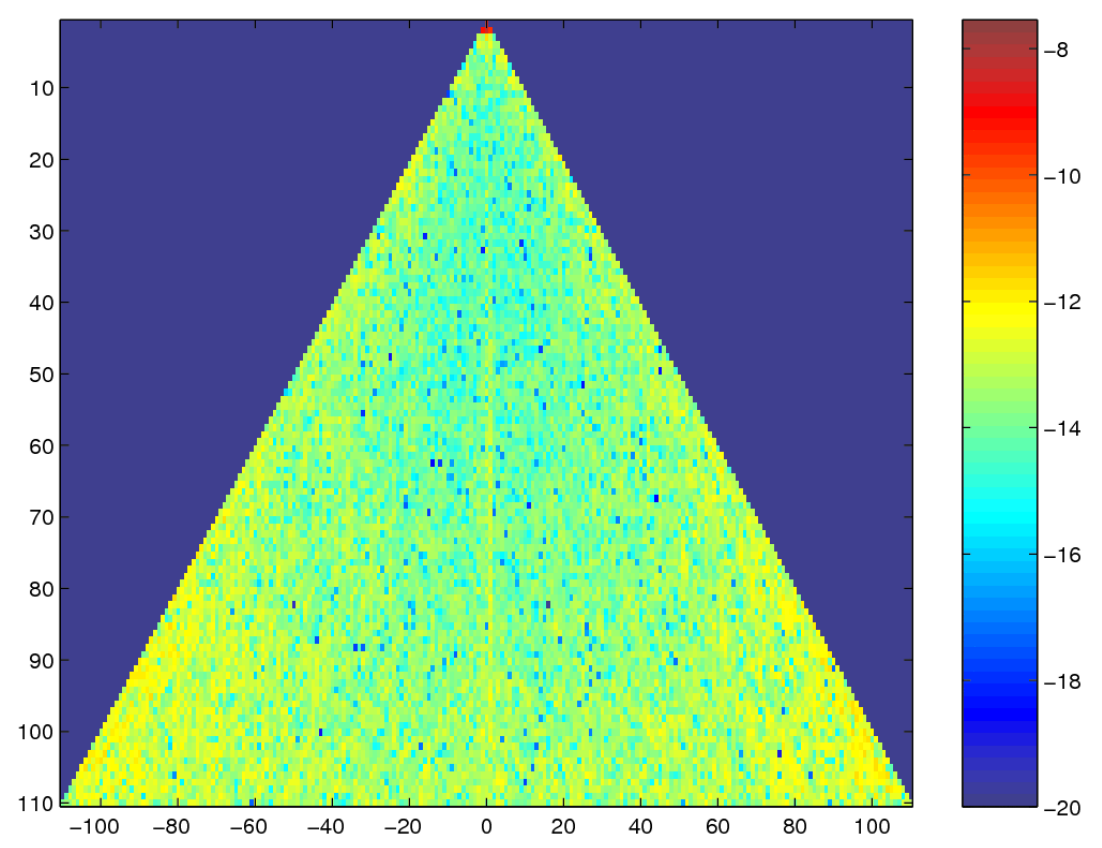

Fig 6. $\mathrm{C}_{\mathrm{nm}}$ (left) and $\mathrm{S}_{\mathrm{nm}}$ (right) coefficient error per degree (top to bottom) and order (mid to side) 0 to 110 for the test scenario presented in Figure 5 (right).

\section{Low-degree coefficients}

Our simulations indicate that in case of $10 \mu \mathrm{m} / \mathrm{s}$ SST accuracy and $1 \mathrm{~m}$ (Weilheim-only) SEST, dimensionless normalized degree- 2 coefficients of the lunar gravity field can be determined at an accuracy of about $0.7 \cdot 10^{-10}$, and degree- 3 coefficients at about $0.3 \cdot 10^{-10}$ after 28days, corresponding to a selenopotential accuracy at LEO orbital altitude of about $1.8 \cdot 10^{-4}$ and $0.9 \cdot 10^{-4} \mathrm{~m}^{2} / \mathrm{s}^{2}$, respectively. This leads us to infer that, after a certain lifetime in orbit, PRARE-L onboard LEO should be able to sense the lunar gravitational body tide amplitude at a given period with an accuracy of few $10^{-4} \mathrm{~m}^{2} / \mathrm{s}^{2}$ or even better. Given that the degreedependent maximum tide-generating potentials of the Earth and Sun amount to $19.5 \mathrm{~m}^{2} / \mathrm{s}^{2}$ (Earth, degree-2, even though the major part of this is absorbed in the permanent lunar tide), $0.086 \mathrm{~m}^{2} / \mathrm{s}^{2}$ (Earth, degree-3), and $0.108 \mathrm{~m}^{2} / \mathrm{s}^{2}$ (Sun, degree-2), and provided that $\mathrm{k}_{2}$ and $\mathrm{k}_{3}$ are on the order of 0.02 and 0.01 , respectively (Sohl, personnel communication), it can be safely expected that PRARE-L onboard LEO will be able to directly measure the low-degree lunar tidal potential Love numbers $\mathrm{k}_{2}$ and $\mathrm{k}_{3}$ (after certain lifetime in orbit). This would be a "First" in Planetary Sciences with important implications not only for the current understanding of the lunar interior but also for terrestrial planet formation and evolution in general (Sohl and Schubert, 2007).

\section{Summary and Outlook}

We have performed a simulation study which shows the anticipated improvement of the lunar gravity field model with respect to the current (LP150Q model) or future (SELENE) gravity field knowledge in the framework of the planned German Lunar Explorations Orbiter (LEO) mission based on PRARE-L Satellite-to-Satellite (SST) and satellite-Earth-satellite (SEST) tracking observations. All results are unbiased solutions obtained without regularization meaning that the PRARE-L instrument will provide a numerically stable and high quality lunar gravity field in only 28 days. Our main result is that the lunar gravity field mean error could be reduced to less than $0.1 \mathrm{mGal}$ at a spatial resolution of $50 \mathrm{~km}$. This is (in the spectral domain) a factor of 10 (long wavelengths) and some 100 (mid- to short-wavelengths) 
improvement relative to predictions made for SELENE, and about a factor of 1000 with respect to the LP150Q model. Also, a higher spatial resolution of up to $28 \mathrm{~km}$ seems feasible, which would be another factor of improvement with respect to SELENE of 2-3. These results can be achieved by using only one SEST terrestrial ground station with $1 \mathrm{~m}$ tracking accuracy and a SST tracking uncertainty of $3 \mu \mathrm{m} / \mathrm{s}$. The latter incorporates $1 \mu \mathrm{m} / \mathrm{s}$ PRARE-L observational noise and $2 \mu \mathrm{m} / \mathrm{s}$ for errors resulting from the transformation from the antenna phase center to the center of mass of the satellites. Less precise SST observations as still assumed during the initial phase-0 simulations caused e.g. by thermal effects, fuel sloshing or high gain antenna or solar panel movements at the main orbiter in case of only one LEO subsatellite, would more or less proportionally diminish the quality of the resultant lunar gravity field solution. Solar radiation errors can be sufficiently adjusted by model scaling factors, or, if implemented, from direct measurements of the RaPS (Radiation Pressure Sensor) instrument, which is a modified version of the Coarse Earth and Sun Sensor (CESS) used for GRACE attitude determination (Doll and Wolters, 1999), and which is intended to observe the global lunar albedo field for the very first time.

The current simulations are based on only one 28 days arc without data gaps. It is expected that, if a longer gravity field mission duration would be realized (at least 1 year is suggested by the LEO science team and seems to be feasible as indicated by the industry studies) the resulting accuracy could be improved by a factor of square root $n$, where $n$ is the number of observed months. Also, the low-degree coefficients of the lunar gravity field would be derived with high accuracy allowing the precise determination of the corresponding lunar Love numbers. Within Phase-A, which started in April 2008, additional simulations taking into account further error sources such as transformation of the SST measurements from the antenna phase center to the center of mass, lunar albedo, transformation errors from Earth to Moon fixed reference frame, data gaps or higher spatial resolutions $(n=200)$ will be performed.

High-precision PRARE-L SEST and a long gravity mission duration, as proposed for LEO, could have another very interesting implication: The largest general-relativistic orbital perturbation for a moon orbiter, as predicted by post-Newtonian theory, would be the gravitoelectric periselenium advance amounting to $8.5 \cdot 10^{-14} \mathrm{rad} / \mathrm{s}$ for LEO, which is due to the lower orbit at $50 \mathrm{~km}$ altitude about 5\% faster compared to SELENE (Iorio 2002), and independent of the orbit inclination. Other general-relativistic precession effects are three orders of magnitude smaller. The gravitoelectric periselenium advance however projects directly onto the along-orbit perturbation, with about $0.37 \mathrm{~m}$ after 28 days and is steadily progressing. Depending on the final sub-satellite orbit accuracy $(1 \mathrm{~m}$ radial error should be feasible as soon as the final lunar gravity field has been derived), this effect might thus become visible after some months of LEO orbiting and would revive the experimental basis of relativistic effects in the Earth-Moon system.

Finally, it has to be noted that PRARE-L could provide, besides the gravity field tracking observations, also very useful signals for time synchronization of e.g. SLR or VLBI ground stations. It is an elegant tool to support future demanding missions with its highly precise phase measurements on code and carrier. It is able to support radio science missions as well as missions involving cold atom or optical clocks in space. The principle can be equally applied to microwave and optical communication links up to interplanetary distance.

Acknowledgement: The authors would like to thank Rune Floberghagen and an anonymous reviewer for their comments and suggestions which helped to improve the paper. 


\section{References}

Bedrich, S., Flechtner, F., Förste, Ch., Reigber, Ch., \& Teubel, A., PRARE System Performance, Proceedings of the 3rd ERS Symposium on Space at the service of our Environment, Florence, Italy, 17-21 March, 1997 (ESA SP-414), 3 Vols., May 1997.

Doll, B., \& Wolters, R., GRACE Coarse Earth and Sun Sensor Specification, GRACE project document 327-40230, issue 1, 1999.

Floberghagen, R., Lunar Gravimetry: Revealing the Far-Side, Astrophysics and Space Science Library, Vol. 273, Kluwer Academic Publishers, Dordrecht/Boston/London, 2002.

Floberghagen, R., Noomen, R., Visser, P., \& Racca, G., Global lunar gravity recovery from satellite-to-satellite tracking, Planet. Space Sci., Vol. 44, No. 10, pp. 1081-1097, 1996.

Foing B.H., MORO - Moon Orbiting Satellite, Phase A Study Report, ESA Doc. SCI(96)1, March 1996.

Iorio, L., Is it possible to test directly general relativity in the gravitational field of the moon?, Class. Quantum Grav. 19, 2393-2398, 2002.

Jaumann., R., Spohn, T., Hiesinger, H., Jessberger, E.K., Neukum G., Oberst J., Helbert, J., Christensen U., Keller, H.U., Mall, U., Böhnhardt, H., Hartogh, P., Glassmeier, K.-H., Auster, H.-U., Moreira, A., Werner, M., Pätzold, M., Palme, H., WimmerSchweingruber, R., Mandea, M., Lesur, V., Häusler, B., Srama, R., Kempf, S., Hördt, A., Eichentopf, K., Hauber, E., Hoffmann, H., Köhler, U., Kührt, E., Michaelis, H., Pauer, M., Sohl, F., Denk, T., \& van Gasselt, S., Lunar Explorations Orbiter (LEO) Vorschlag für eine wissenschaftliche Mondmission, DLR, 31.01.2007a.

Jaumann, R., Spohn, T., Hiesinger, H., Jessberger, E.K., Neukum G., Oberst J., Helbert, J., Christensen U., Keller, H.U., Mall, U., Böhnhardt, H., Hartogh, P., Glassmeier, K.-H., Auster, H.-U., Moreira, A., Werner, M., Pätzold, M., Palme, H., WimmerSchweingruber, R., Mandea, M., Flechtner, F., Lesur, V., Häusler, B., Srama, R., Kempf, S., Hördt, A., Eichentopf, K., Hauber, E., Hoffmann, H., Köhler, U., Kührt, E., Michaelis, H., Pauer, M., Sohl, F., Denk, T., \& van Gasselt, S., LUNAR EXPLORATION ORBITER (LEO): PROVIDING A GLOBALLY COVERED, HIGHLY RESOLVED, INTEGRATED, GEOLOGICAL, GEOCHEMICAL, AND GEOPHYSICAL DATA BASE OF THE MOON, Abstract for Lunar Exploration Analysis Group, Conference "Enabling Exploration: The lunar outpost and beyond; Houston, Oct. 1.-5., $2007 \mathrm{~b}$.

Kaula, W.M., The investigation of the gravitational fields of the Moon and planets with artificial satellites, Adv. Space Sci. Technol., 5, 210-230, 1963.

Konopliv A.S., Asmar, S.W., \& Yuan, D.N., Recent gravity field models as a result of the Lunar Prospector mission, Icarus, 150, 1-18, 2001.

Lemoine, F.G.R., Smith, D.E., Zuber, M.T., Neumann, G.A., \& Rowlands, D.D., A 70th degree lunar gravity model (GLGM-2) from Clementine and other tracking data, J. Geophys. Res. (Planets), 102 (E7), 16339-16359, 1997.

Matsumoto, K., Hanada, H., Namiki, N., Iwata, T., Goossens, S., Tsuruta, S., Kawano, N., \& Rowlands, D., A simulation study for anticipated accuracy of lunar gravity field model by SELENE tracking data, J. Adv. Space Res., 42, 2, doi:10.1016/j.asr.2007.03.066, 2008.

Reigber, Ch., Schmidt, R., Flechtner, F., König, R., Meyer, U., Neumayer, K. H., Schwintzer, P., \& Zhu, S. Y., An Earth gravity field model complete to degree and order 150 from GRACE: EIGEN-GRACE02S, J. Geodyn., 39, 1-10, 2005.

Schäfer, W., Kufner, M., Siccardi, M., Feltham, S., Aguilar Sanches I., Seidel, A., Hess, M.P., Bedrich, S., \& Flechtner, F., Pseudo-Noise based Ranging and Time-Transfer Systems: PRARE, ACES-MWL and beyond, Proceedings ESA $4^{\text {th }}$ International Workshop on 
Tracking, Telemetry and Command Systems for Space Applications, Paper 827402, 11 - 14 September 2007, ESOC, Darmstadt, Germany, 2007.

Sohl, F., \& Schubert, G., Interior structure, composition, and mineralogy of the terrestrial planets, Treatise on Geophysics (Editor-in-Chief G. Schubert), Volume 10, Planets and Moons (Ed. T. Spohn), 27-68, 2007.

Standish, E. M., JPL planetary and lunar ephemeredes DE405/LE405, IOM 312, Jet Propulsion Laboratory publication F-98-048, Pasadena/CA, 1998.

Tapley, B.D., \& Reigber, Ch., The GRACE Mission: Status and Future Plans, EOS Trans. AGU, 82(47), fall Meeting, Suppl. G14C-02, 2001.

Wiezcorek, M.A., The gravity and topography of terrestrial planets, Treatise on Geophysics (Editor-in-Chief G. Schubert), Volume 10, Planets and Moons (Ed. T. Spohn), 165-206, 2007.

Williams, J.G., A scheme for lunar inner core detection, Geophys. Res. Lett., Vol. 34, L03202, doi:10.1029/2006GL028185, 2007.

Zuber, M.T., Smith, D.E; Lemoine, F.G., \& Neumann, G.A., The shape and internal structure of the Moon from the Clementine mission, Science, 266 (5192), 1839-1843, 1994. 